common symptoms. A small number of cases which had commenced as sciatica had subsequently developed definite affection of the hip-joint. Swelling of the joint and increased heat in its neighbourhood were seldom observed, owing no doubt to the deep situation of the joint and to the chronicity of the affection. On the whole Dr. Bruce must be given the credit of making a strong case in favour of his theory of the cause of so called sciatica.

$$
1 \text { Lancet, Aug. } 22 .
$$

\section{PRACTICAL POINTS IN THE TREATMENT} OF RHEUMATISM.

The virtues of salicin and the salicylates in the treatment of rheumatic fever have been so generally recognised that the older methods of treatment are often entirely overlooked. Nevertheless, there were brave men before Agamemnon, and a parallel remark may be made in reference to the salicylates. There are still physicians who hold fast to the alkaline treatment of acute rheumatism, and without instituting any invidious comparisons it can be safely said that it is a therapeutic method of distinct value. A not infrequent cause of failure is the fact that the alkaline salts are not given in sufficiently large doses. To succeed, it is absolutely essential to order the potassium citrate, bicarbonate, etc., in such amounts as to produce and maintain an alkaline reaction in the urine. The doses necessary to effect this vary with the individual patient, but speaking generally, it will be found that doses considerably in excess of those commonly prescribed are needed to change the normal reaction of the urine. The test of sufficiency is readily applied, and the physician can never be sure that he is adopting the alkaline treatment in a thorough and efficient fashion unless he makes sure that his remedies are modifying the urine in the manner indicated. In many sub-acute cases the alkaline treatment, when fully practised, will be found to succeed when other well-known remedies have failed. It is easy to associate it with quinine when a tonic influence is required, and the best way is to add the quinine to each dose of the alkaline mixture at the time of administration. The quinine, of course, does not dissolve, and the patient is thus spared the bitter taste of the remedy. Chronic rheumatism, it is generally said, does not yield to the salicylates, and certainly the chronic, painful enlargements and deformities of the joints variously termed rheumatic or rheumatoid arthritis are undoubtedly very resistant to all forms of treatment. Yet sodium salicylate is not without virtue in this condition. Its special influence is to lessen the stiffness and discomfort which the patient experiences when he wakes in the morning. This is very often a cause of considerable disability, and it is in many instances much improved by sodium salicylate given in a single full dose at bed time. The patient may commence, say, with 15 or 20 grains, and this should be steadily increased until either relief is obtained or physiological evidences of the action of the salicylate are produced. The remedy can be continued for weeks or months and frequently with great advantage. A local application of great service in the same disease is a strong liniment of capsicum. This should be sprinkled on flannel which is to be wrapped round the affected joints and worn all night. Menthol is another remedy which often gives prompt relief to rheumatic pains, more especially when these affect the muscles. The best method of applying it is in the form of an ointment. made with equal parts of lanoline and vaseline. The proportion of menthol ordered should be a drachm in an ounce of the ointment. The greasy basis permits the use of energetic friction and also favours the passage of the menthol through the epidermis.

\title{
PROGRESS IN ORTHOPAEDIC SURGERY.
}

The Surgery of the Paralyses of Children.-Robert Jones $^{1}$ has given an instructive address on this subject, in which he deals with infantile paralysis and spastic paralysis. In infantile paralysis he urges that the aim of the surgeon should be to prevent deformity, as well as to cure it, when it has supervened. The factors in the production of deformity are gravity, body-weight, the shape of the articular facets, and unbalanced muscular action ; and he illustrates his proposition by concrete examples in the cases of the foot, the knee, and the hip. He further thinks that surgery has been too long silenced by the pathological reports of these cases, and that we are too ready to infer that because some of the motor cells are damaged, the muscles, either as groups or entities, are hopelessly destroyed. Extended clinical experience proves that this is not so, for muscles which are apparently entirely paralysed will recover often to a very considerable degree when placed in suitable conditions. And this fact is the keynote of the surgical treatment of infantile paralysis. It is best illustrated by dropped wrist from paralysis of the extensors. Recovery will never take place so long as the weakened extensors are continuously stretched by allowing the wrist to droop. If the hand is kept hyper-extended, and the extensor muscles are suitably massaged, recovery soon begins to be manifest, and will be complete in most cases in 18 months to two years. By this treatment the lengthened extensor muscles are shortened, and the contracted flexors are lengthened, and thus the muscular balance is restored. Tendon transplantation is of the utmost value in the treatment of paralytic deformities, because it is scientific in its methods and easy and safe in its application. It is scientific because it aims at distributing around a joint in an equal manner the residue of muscular power. Or to put the matter more clearly, and to take a definite example : if the foot is in a position of equino-valgus it is quite evident that the tendo Achillis and peronei are exerting an undue power, and that this power is running to waste, while the invertors and dorsiflexors are unable to oppose them. The scientific method of transplantation will transfer part of the force in the tendo Achillis to the invertors, and part of the power of the evertors into the invertors, thus obtaining good muscular balance around the ankle. This is a more rational proceeding than the old practice of dividing the tendons of the healthy muscles and then trying to maintain the foot in good position by apparatus, which must be worn for a life-time. In the treatment of spastic paralysis 
equally great advances have been made. Every contracted tendon and muscle should be divided, the limbs straightened, and all flexor deformities fully overcome, and then, this being highly important, the ehild must be educated to use his limbs properly.

Tendon Transplantation for Infantile Paralysis. W. P. Montgomery ${ }^{2}$ gives accounts of 25 cases in which this operation was done to remedy the sequelæ of anterior poliomyelitis. After reviewing the history of the operation, Montgomery details four classical methods, viz. : (1) Section of the tendon of a healthy muscle and its implantation into the tendon of a paralysed one; (2) the "passive" method of Hoffa, which consists in section of the tendon of a paralysed muscle and its attachment to an acting undivided muscle; (3) the transference of a slip from an active muscle tendon to a paralysed one ("active" method of Hoffa); (4) the attachment of an active muscle or tendon, or of a slip from it, to the periosteum at a new site. Montgomery has employed all these methods in his cases. There are at least nine other methods in vogue, to which allusion might well have been made. Certain conclusions are deduced from the cases. These are briefly : (1) If the acting and paralysed muscles are the more nearly allied in their actions, the improvement is much more rapid and the earlier the results will appear; (2) the greater the length of the tendon of a paralysed muscle to which the acting muscle is applied, the less satis. factory the earlier result and the slower the improvement will be ; this is due to stretching of the paralysed tendon; (3) the results of the passive method, division of the paralysed tendon and its suture to the intact acting one are much inferior to those of the active method, in which a slip is taken from the normal tendon; (4) there is no doubt that at the time of transplantation all deformity must be corrected; (5) as regards after-treatment, the ideal method is to begin massage and passive movements after three weeks and gentle active movements after four weeks. The material used for tendon grafting was silk, and in no cases did any stitch abscesses arise.

Snap or Trigger Finger.-H. L. Barnard ${ }^{3}$ records four cases of this interesting and not uncommon condition. In one case on moving the finger painful nodules could be felt slipping up and down, and these nodules were in the flexor sublimis tendon; and in another instance where the thumb was affected the nodules were on the flexor longus pollicis tendon, and in two other cases painful nodules could be felt wedged in between the sesamoid lines of the short flexor of the thumb. It is pointed out that the majority of cases have occurred in women, and most of them are over 14 years of age. The snapping is due to changes in the tendon, in the tendon sheath, and in the joint which snaps. As to treatment, many of the cases are evanescent; and for such, rests on a splint with pressure from a pad placed over the swelling, and later on massage, appear to be suitable and successful forms of treatment. But if a nodule can be felt and it persists for some time, the right course to take is to expose the tendon and remove the nodule or lessen the disproportion between the tendon and its sheath. If synovial fringes exist they should be removed. F. Griffith ${ }^{4}$ also describes a case, which affected the middle and ring fingers in a woman. In an analysis of 121 cases, Necker found either acute or chronic rheumatism in 52 ; traumatism in 13 ; occupation causes in 47 ; congenital conditions in two cases, and in seven no cause could be assigned. Griffith is in accord with Barnard as to the causation and treatment. W' $\mathrm{W}^{\top}$ iteford ${ }^{5}$ records a case in a man aged 39 who had become an enthusiast at golf. The left little finger was affected. With precautionary measures the affection subsided and operation was unnecessary.

Forcible Rectification of the Spine for Pott's Disease.-J. V. Young ${ }^{6}$ describes a case under his treatment at Beyrout in Syria, and alludes to the work of Tubby and Jones ${ }^{7}$ in this direction. Young followed their procedure almost exactly. He found the operation safe and easy, and was pleased with the immediate result. Now that some years have elapsed since this treatment was commenced, there is no doubt that if cases be properly cared for afterwards there is lasting improvement.

1 Lancet, Feb. 14, 1903, p. 422. ${ }^{2}$ Med Chron., November, 1902, p. 97. 3 Practitioner, February 1903, p. 178. 4 Anns. of Surg., October 1902, p. 586. 5 Brit. Med. Jour., Jan. 31. 1903, p. 251 . 6 New York Med. Rec., August 1902, p. 173. 7 Med. Annual, 1898, and Clin. Soc. Trans, 1899 and 1901.

\section{PROGRESS IN GYNAECOLOGY.}

Primary Cancer of the Fallopian Tube--Primary cancer of the Fallopian tube is such a comparatively rare disease that every case is worthy of careful study. Graefe ${ }^{1}$ records a case which occurred in a sterile woman aged 51 ; she had been married 22 years, and apparently had hitherto been free from any pelvic inflammatory disease. The periods, regular till nearly one year before observation, became very profuse, and seemed to reappear after an interval of a week. On vaginal examination a tense sausageshaped tumour was felt in Douglas' pouch. The patient declined any operation. Two and a half years later she returned to Graefe, when he found that the tumour in Douglas' pouch had not altered in size, but he was able to detect a rounded tumour the size of the fœtal head to the right of the one in Douglas' pouch. He opened the abdomen and removed a cyst of the right ovary which had burrowed deeply into the right broad ligament, and a distended right tube shaped like a tobacco pipe. On opening this he found a papillary tumour the size of a chestnut growing from the inner wall of the ampullary portion of the tube. This proved to be a malignant growth ; the epithelium of the villous masses was arranged in several layers, and some of it had invaded the deeper tissues in the tubal wall. The patient was free from recurrence eight months after the operation. The history clearly showed that a papilloma of the tube, which must have been present for some time, had become malignant. Bland-Sutton ${ }^{2}$ also records a case in which the disease arose in the tubal ampulla near its ostium, and there was an unusually long unimplicated stretch of tube between it and the uterine 